\title{
SUMMARY OF THE "DIFFRACTION AND VECTOR MESONS" WORKING GROUP AT DIS06
}

\author{
H. LIM \\ Agonne Natonal Laboratory, Argonne, Illinois 60439-4815, USA \\ L. SCHOEFFEL \\ DAPNIA/Service de physique des particules, CEA/Saclay, 91191 Gif-sur-Yvette \\ cedex, France \\ M. STRIKMAN \\ Penn State University, University Park, PA 16801, PA, USA \\ We survey the contributions presented in the working group "Diffraction and Vec- \\ tor Mesons" at the XIV International Workshop on Deep Inelastic Scattering.

\section{Introduction} \\ Studies of diffractive processes provide a much more detailed information \\ about the pattern of the high energy strong interactions than inclusive cross \\ section. One can infer from these studies both the transverse range of the \\ interaction as well as its intensity. The knowledge of the strength of the \\ interaction as a function of the impact parameter provides a direct infor- \\ mation on a possible proximity of interaction of hadrons or small dipoles \\ with nucleons to the black disk limit (BDL) of the maximal strength of the \\ interaction. The BDL is especially interesting for the case of the interac- \\ tion of the small objects as it corresponds to the situation when the strong \\ coupling constant is small but the expansion over the twists breaks down.
}

\section{Diffraction and factorization}

In hadron-hadron (or lepton-hadron) scattering a substantial fraction of the total cross section is due to diffractive reactions. In elastic $p \bar{p}$ scattering, both projectiles emerge intact in the final state, whereas single or double diffractive dissociation corresponds to one or both of them being scattered 
into a resonance or continuum state with mass, $M_{X} \ll \sqrt{s}$. The two groups of final-state particles are well separated in phase space and in particular have a large gap in rapidity between them. Similar features hold for leptonhadron scattering with $l+p \rightarrow l+X+Y$ observed at HERA, the low-mass excited state $Y$ carrying the same quantum numbers (except spin) as the incident proton.

Then, soft diffractive interactions follow specific properties which find a natural description within Regge theory, in which they can be interpreted as mediated by the leading singularity in the angular momentum plane with vacuum quantum numbers, called Pomeron.

In the perturbative regime Pomeron like behavior of the elastic amplitude emerges at high energies due to the exchange of a ladder of gluons and quarks in t-channel. In difference from the universal Pomeron of the Regge theory perturbative Pomeron is a resolution dependent construction which is not directly connected to the nonperturbative Pomeron.

Following the above considerations, it is clear that diffractive events (at HERA) can be selected by (at least) three methods: (a) by the requirement of the existence of a large rapidity gap (LRG) between the produced hadronic system $\mathrm{X}$ and the outgoing hadron, (b) by tagging leading baryons which carry a large fraction of the incoming hadron beam energy, and (c) using the $M_{X}$ method which is based on the different characteristics of the $M_{X}$ distributions in diffractive and non-diffractive processes according to the Regge model (see Fig. 1). Using this model for hard

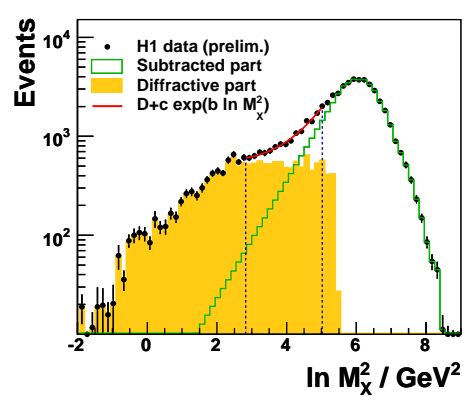

Figure 1. $\ln M_{X}^{2}$ spectra with the different contributions : diffractive plateau (full histogram) and high mass peak due to non-diffractive events. processes which are superposition of interactions with hard and soft Pomeron may potentially introduce an uncertainty in the analysis. Also, LRG method and proton tagging are commonly used at the TEVATRON to characterize a diffractive event.

Note that samples defined in these ways also contain processes for which nondiffractive mechanisms become important which one can try to model via contribution of subleading Regge trajectories, while the $M_{X}$ method suppresses their contributions. Also, tagging the outgoing hadron presents the interesting advantage of excluding background from dissociative pro- 
cesses, $l+p \rightarrow l+X+Y$, with the drawback that the acceptance and then kinematic coverage are quite limited for this technique.

Following arguments related to the color transparency phenomenon in QCD, one can prove the QCD factorization theorem for semi inclusive processes $l+p \rightarrow l+X+h$ for fixed Feynman $x_{L} \equiv 1-x_{\mathbb{P}}$ and transverse momentum of the hadron ${ }^{1}$. In most of the data analyses this theorem is supplemented by an assumption motivated by the Regge model that the diffractive (for small $\mathrm{x}$ ) parton densities (DPDFs) can be factorized as a product of the function of $x_{\mathbb{P}}, p_{t}$ and a function of $\beta=x / x_{\mathbb{P}}, Q^{2}$. Then, diffractive parton density functions (DPDFs) integrated over $p_{t}$ can be extracted from the measurements of diffractive structure function $\left(F_{2}^{D}\right)$ and can be used to predict the rates of diffractive jet or diffractive heavy flavour processes. Though the DPDFs extracted from HERA data overestimate the rate of diffractive dijet process at the TEVATRON, it could be explained by absorptive effects since the interaction of protons at small impact parameters is practically black and cannot lead to diffraction. Similar suppression effect is an essential ingredient of the preditions on exclusive diffractive Higgs production at the LHC.

\subsection{Leading neutron measurement}

\section{ZEUS}

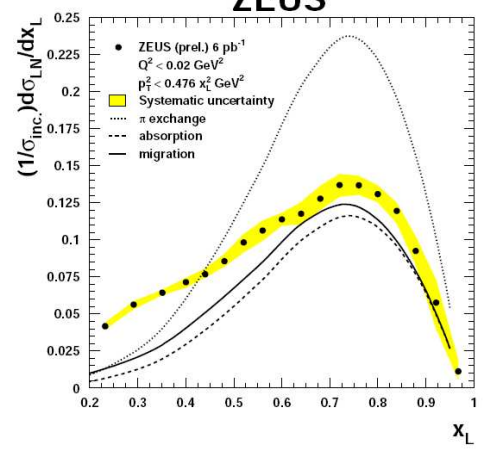

ZEUS

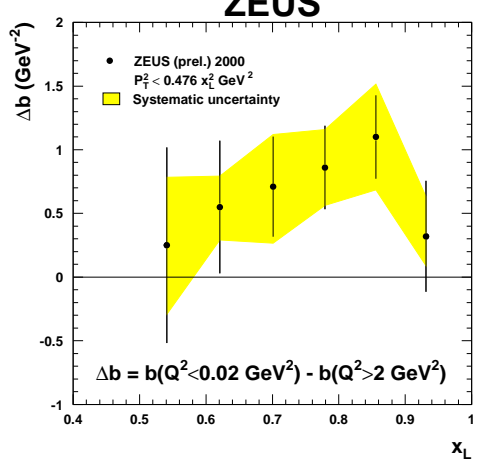

Figure 2. (left) Leading neutron energy spectra for the photoproduction sample. Curves are from the predictions from the one-pion exchange model including the effects of neutron absorption. (right) The difference between slopes in photoproduction and DIS vesus $x_{L}$.

The production of leading baryons in ep scattering has been studied at the HERA collider. The ZEUS Collaboration has presented recent mea- 
surement of the energy distributions of leading neutrons in $e p$ by real and virtual photons ${ }^{2}$ for which in the DIS limit the QCD factorization theorem ${ }^{1}$ should be valid. The ZEUS forward neutron calorimeter (FNC) measures neutrons which carry a large fraction, $x_{L}$ of the incoming proton beam energy and are produced at a small angle with respect to the incoming proton direction. Measurements are consistent with the expectation that multiPomeron exchanges which lead to absorption effects decrease with increase of $Q^{2}$. Data also indicate that the neutron multiplicities are pretty similar for the case of the processes dominated by scattering off quarks and off gluons (dijet production) which is consistent with the fragmentation scenario of ${ }^{3}$.

The DIS data were described in the one-pion exchange model including effects of absorption ${ }^{4}$ (see Fig. 2(left)). The $p_{T}^{2}$ distributions of neutrons were parametrized using $\exp \left(-b p_{T}^{2}\right)$. Though the tendency of $b$ as a function of $x_{L}$ is similar between the theoretical predictions and data, none of the models describes the data in scale. In Fig. 2 (right), the slopes of photoproduction samples is clearly larger than those of DIS samples. However the model assumes that the t-slope of the $\pi N N$ vertex is close to zero which is contradiction with the analyses of the antiquark distributions in nucleons, see a review in ${ }^{5}$. Also the model leads to much smaller rates of the neutron production for the dijet trigger.

\subsection{Inclusive diffractive measurements}

H1 has presented the measurements of diffractive reduced cross sections covering a wide kinematic range and obtained with the proton-tagging $\operatorname{method}^{6}$, the LRG method ${ }^{7,8}$ and the $M_{X}$ method $^{8}$. Note that the reduced cross section is equal to the diffractive structure function $F_{2}^{D(3)}$ except for the largest values of the inelasticity variable $y$, where a correction taking into account the effect of the longitudinal component has to be considered. In Ref. ${ }^{8}$, the $\mathrm{H} 1$ experiment has compared cross section values based on a LRG selection and a $M_{X}$ method, all together compared with ZEUS published results ${ }^{9}:$ in the range of the comparison (medium $M_{X}$ vlaues), a good agreement is observed for all these data sets and methods.

\subsection{Semi-inclusive diffractive measurements}

The QCD factorization theorem predicts that the same DPDFs should describe both inclusive DIS diffraction and the semi-inclusive processes like dijet or charm production. The analyses of the data clearly show that 


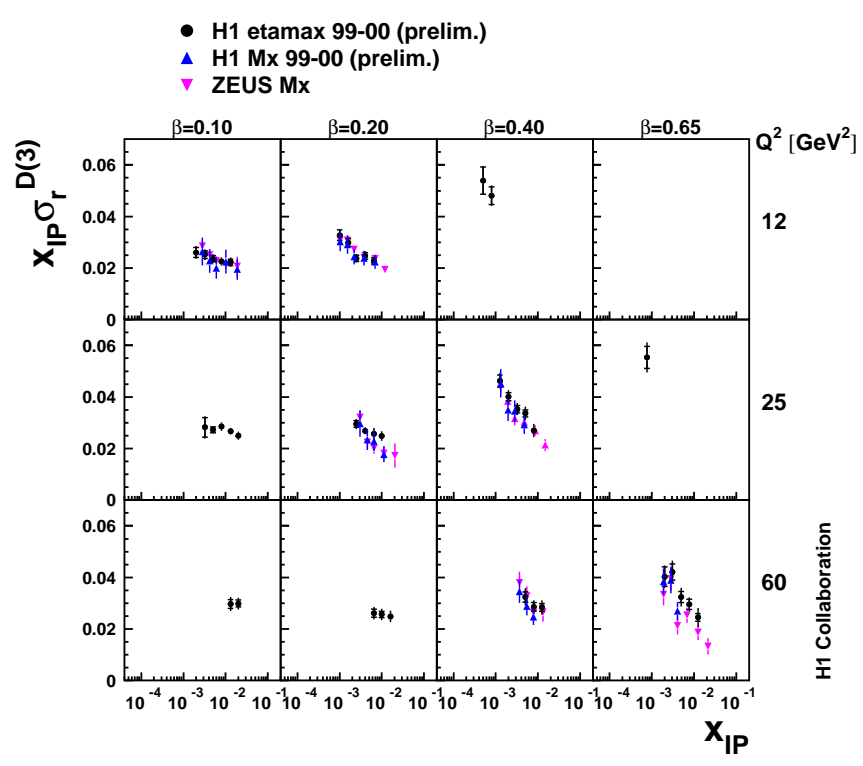

Figure 3. Comparisons of the diffractive reduced cross sections obtained using the LRG and $M_{X}$ methos with 1999-2000 data and with measurements from the ZEUS Collaboration.

the gluon density largely dominates the quark content of the diffractive exchange ${ }^{7,10,11}$.

Several issues have been discussed on such procedures ${ }^{10,11}$ concerning the proper Regge factorisation implementation and relative importance of the leading twist and higher twist contributions in particular due to the interaction of the small size dipoles (which dominate in high $Q^{2}$ exclusive diffraction).

In particular, the approach described in Ref. ${ }^{11}$ does not assume Regge factorization and suggests that the collinear factorization theorem, though valid asymptotically in diffractive DIS, has important modifications at the energies relevant at HERA and predicts a significantly larger value of $\alpha_{\mathbb{P}}(0)$ in diffraction than in soft processes.

The new data reported at the meeting seem to suggest that the effective Pomeron trajectory for diffraction has an intercept $\alpha_{\mathbb{P}}(0)=1.118 \pm$ 0.008 (exp. $)_{-0.010}^{+0.029}$ (model) which is close to the one for the nonperturbative Pomeron.

The comparison of semi-inclusive final states, as diffractive dijets at HERA ${ }^{12,13}$ and inclusive data provides now a convincing confirmation 
of the QCD factorization for $\beta \leq 0.4$. For larger $\beta$ the uncertainties in extraction of the gluon DPDF appear to be too large to reach definite conclusions about consistency of the gluon densities extracted from DIS and from semi-inclusive processes.

Fig 4 presents the gluon distribution of the diffractive exchange extracted by combining the inclusive and dijet measurements from the $\mathrm{H} 1$ experiment, which leads to a much better constraint on the gluon density at large momentum fraction. At TEVATRON also, dijet events in double Pomeron exchange (DPE) show a strong sensitivity to diffractive gluon density 10,14 and new experimental results have been presented ${ }^{15}$ in terms of the dijet mass fraction. This variable $\left(R_{j j}\right)$ is defined as the dijet invariant mass $\left(M_{j j}\right)$ divided by the mass of the entire system $M_{X}$, calculated using all available energy in the calorimeter. If jets are produced exclusively, $R_{j j}$ should be equal to one. Data are compared with MC expectations on Fig. 5. At large $R_{j j}$ values, the excess of events in the data with respect to inclusive DPE dijet production, which is described by POMWIG ${ }^{16} \mathrm{MC}$, is well accounted for by the DPEMC ${ }^{17} \mathrm{MC}$ sample of exclusive events (Fig. 5, left).

Another process illustrated in Ref. ${ }^{15}$ concerns exclusive diphoton events, $p \bar{p} \rightarrow p \gamma \gamma \bar{p}$. The final state is cleaner than in exclusive dijet production as hadronization effects are absent, but the expected cross section is smaller.

\section{Exclusive final states}

The dynamics of diffractive interactions can also be studied through exclusive vector meson $\left(\rho^{0}, \omega, J / \psi, \ldots\right)$ and photon production, $l+N \longrightarrow$ $l+V+Y$, where $Y$ is defined as in previous sections as an elastically scattered nucleon or a low-mass dissociative state. At low transverse momentum transfer at the nucleon vertex, the photoproduction of $\rho^{0}, \omega$ and $\phi$ mesons is characterized by a "soft" dependence of their cross-sections in the $\gamma p$ center-of-mass energy, $W$. This can be interpreted in the framework of Regge theory as due to the exchange of a "soft" Pomeron $(\mathbb{P})$ resulting in an energy dependence of the form $\mathrm{d} \sigma / \mathrm{dt} \propto \mathrm{W}^{4\left(\alpha_{\mathrm{P}}(\mathrm{t})-1\right)}$, where the Pomeron is parametrised by a Regge trajectory.

The H1 collaboration has performed a new cross section measurement of the exclusive $\rho^{0}$ photoproduction, using a sample of more than $240 \mathrm{k}$ events recorded in the year 2005. This measurement has been done in the kinematic domain $20<W<90 \mathrm{GeV}$ and $|t|<3 \mathrm{GeV}^{2}$, leading to a determi- 

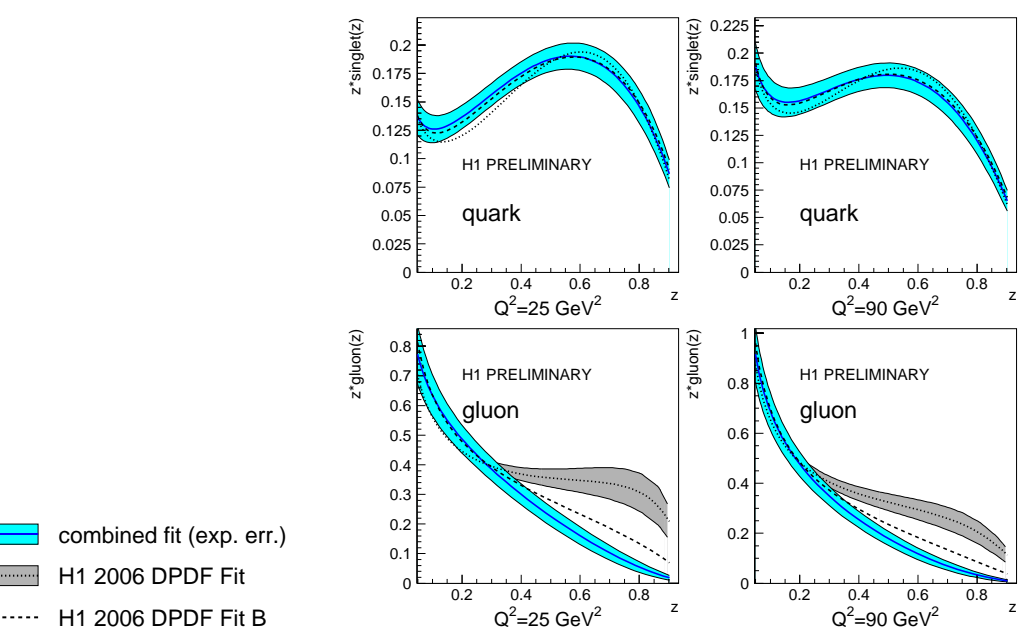

Figure 4. The diffractive singlet density (top) and diffractive gluon density (bottom) for two values of the hard scale $\mu: 25 \mathrm{GeV}^{2}$ (left) and $90 \mathrm{GeV}^{2}$ (right). The blue line indicates the combined fit, surrounded by the experimental uncertainty band in light blue. For comparison, the two dashed lines show two fit results from the inclusive cross sections alone.
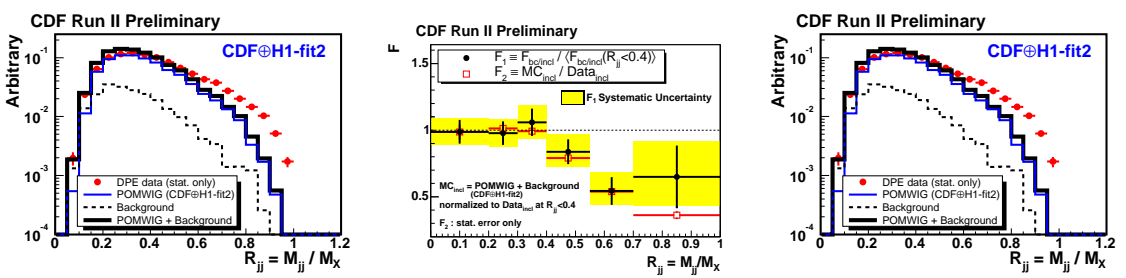

Figure 5. Left: dijet mass fraction in DPE data (points) and best fit (solid) obtained from POMWIG MC events (dashed) and exclusive dijet MC events (shaded); Center: normalized ratio of heavy flavor jets to all jets as a function of dijet mass fraction. Right: $R_{j j}$ distribution for the data (points) and POMWIG MC prediction (thick histogram), composed of DPE dijet events (thin) and non-DPE events (dashed). Data and MC are normalized to the same area.

nation of the $W$ dependence of the cross section in eight bins of $t^{18}$.

Assuming that the Pomeron trajectory is linear they find: $\alpha_{\mathbb{P}}(t)=$ $\alpha_{\mathbb{P}}(0)+\alpha^{\prime} t \simeq 1.093+0.116 t$, which is corresponds to a factor $\sim 2$ smaller $\alpha^{\prime}$ than measured in the $p p$ elastic scattering. However the data do not exclude a possibility that $\alpha^{\prime}$ is about the same for small $\mathrm{t}$, but that effective Pomeron trajectory is nonlinear. Note also that the data use a Regge factorization model to subtract the inelastic background. This assumption 
is expected to be violated at sufficiently large t where pQCD contribution to inelastic diffraction becomes important.

The QCD factorization theorem is valid for production of vector mesons by a large $Q^{2}$ longitudinally polarized photons, and for production of mesons build off heavy quarks ${ }^{19}$. Also, it is often assumed that perturbative QCD is valid if the "hard scale" is provided by the momentum transfer $|t|$ or highly virtual transversely polarized photon or of the vector meson mass, perturbative QCD is expected to apply. In this approximation, diffractive vector meson production can be seen in the nucleon rest frame as a sequence of three subprocesses well separated in time: the fluctuation of the exchanged photon in a $q \bar{q}$ pair, the hard interaction of the $q \bar{q}$ pair with the nucleon via the exchange of (at least) two gluons in a color singlet state, and the $q \bar{q}$ pair recombination into a real vector meson. This approach results in a stronger rise of the cross section with $W$ than for soft processes, which reflect the rise at small $x$ of the gluon density in the nucleon. Furthermore, to take into account the skewing effect, i.e. the difference between the proton momentum fractions carried by the two exchanged gluons, one has to consider generalized parton distributions (GPDs). All these aspects have been covered with new results and are summarised in the following.

The $\mathrm{H} 1$ collaboration has finalized a determination of the diffractive production of $\rho^{0}$ mesons at large $|t|\left(1.5<|t|<10.0 \mathrm{GeV}^{2}\right)$, using data taken during the year 2000, with an integrated luminosity of $20.1 \mathrm{pb}^{-1} 18$. Several spin density matrix elements (SDME), which carry information on the helicity structure of the production amplitudes, have been extracted from the production and decay $\rho^{0}$ angular distributions. The data indicate a violation of the $s$-channel helicity conservation (SCHC), with contributions from both single and double helicity flip being observed. This observations contrast to what was observed for high $|t| J / \psi$ production and is generally attributed to differences in the wave function between $\rho$ and $J / \psi$. The cross section, differential in $t$, for $1.5<|t|<10.0 \mathrm{GeV}^{2}$, is also in reasonable agreement with pQCD models, inspired by the BFKL phenomenology. However these models are based on the leading order BFKL approximation which is known to differ strongly from resummed BFKL. Also none of the available models is able to explain behavior of all spin observables.

New developments on dipole models based on the saturation approach to describe the dipole-proton cross section have been presented ${ }^{23}$. They provide a very good fit for the cross sections of all exclusive processes (in the DIS regime), differential in $Q^{2}, W$ or $t$ (see Fig. 6 and Fig. 7).

One of the key perturbative QCD predictions was universality of the 
slopes (defined on the exponential fit to the differential cross section: $d \sigma / d t \propto \exp (-b|t|)$ at small $t)$ in the leading twist limit ${ }^{24}$. The data shown in Figure 8 are consistent with onset of such universality. decreases with increasing scale $Q^{2}+M_{V}^{2}$ The dipole model is well suited to estimate the onset of the scaling regime by estimating contribution to the slope of the finite size of the $q \bar{q}$ dipole. The rate of convergence of the slopes is consistent with predictions of ${ }^{25}$, see the lines in figure 8 .
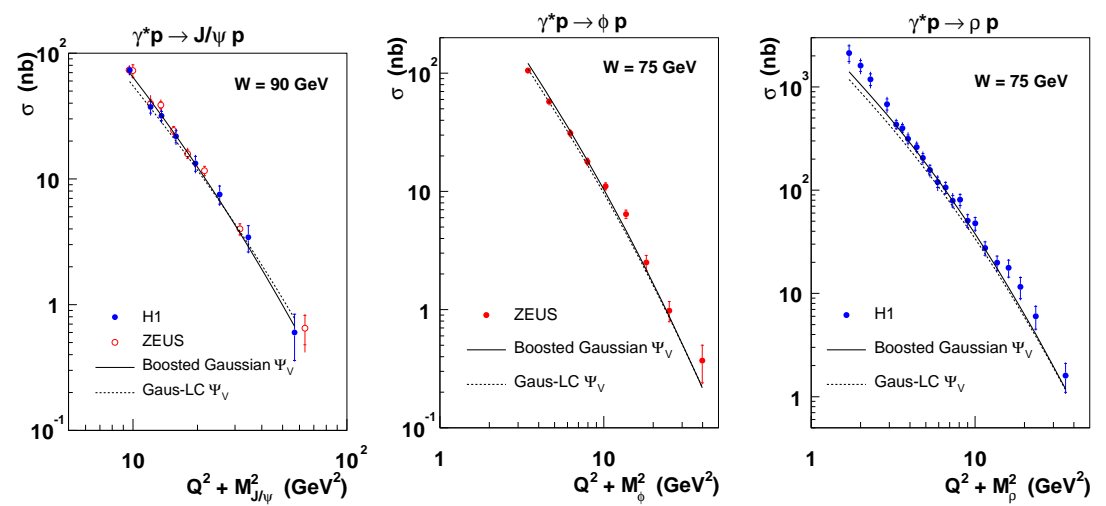

Figure 6. Total vector meson cross section $\sigma$ vs. $\left(Q^{2}+M_{V}^{2}\right)$ compared to predictions from the b-Sat model using two different vector meson wave functions.
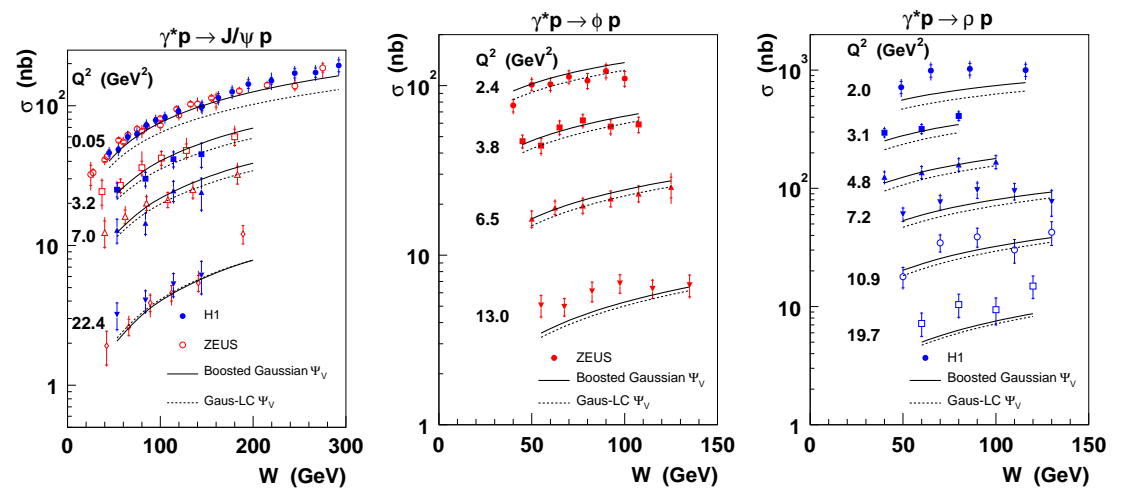

Figure 7. Total vector meson cross section $\sigma$ vs. $W$ compared to predictions from the b-Sat model using two different vector meson wave functions. 


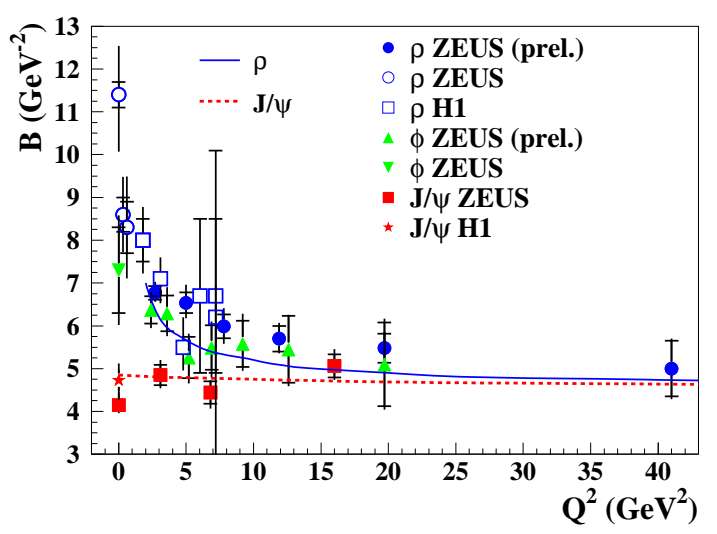

Figure 8. The logarithmic slope of the $t$ dependence at $t=0$ for different meson production channels.

A Fourier transform from momentum to impact parameter space readily shows that $b$ is related to the typical transverse distance between the colliding objects, see ${ }^{21}$ and references therein. At high scale, the $q \bar{q}$ dipole is almost point-like, and the $t$ dependence of the cross section is controlled by the $t$ dependence of the generalized gluon distribution, or in physical terms, by the transverse extension of the gluons in the proton for a given $x$ range.

The COMPASS experiment has presented a study of the diffractive elastic leptoproduction of $\rho^{0}$ mesons, $\mu+N \longrightarrow \mu+\rho^{0}+N$, where $N$ is a quasi-free nucleon from any of the nuclei of their polarized target, at $<W>=10 \mathrm{GeV}$ for a wide range of $Q^{2}, 0.01<Q^{2}<10 \mathrm{GeV}^{2}{ }^{20}$. The COMPASS data provide a large statistics which allows to extend the previous measurements of the $r_{00}^{04}$ matrix element towards low $Q^{2}$. Then, if one assumes SCHC between the exchanged photon and the $\rho^{0}$ meson, one can obtain the ratio $R$ between the longitudinal $\left(\sigma_{L}\right)$ and the transverse $\left(\sigma_{T}\right)$ cross sections (see Fig. 9).

Information about GPDs in lepton nucleon scattering can be provided by measurements of exclusive processes in which the nucleon remain intact. A complete overview of the topic has been presented in Ref. ${ }^{21}$. In particular, the simplest process sensitive to GPDs is Deeply Virtual Compton Scattering (DVCS), i.e. exclusive photon production off the proton $\gamma^{*} p \longrightarrow \gamma p$ at small $|t|$ but large $Q^{2}$, which is calculable in perturbative QCD. Such a final state also receives contributions from the purely electromagnetic Bethe-Heitler process, where the photon is radiated from the 
lepton. The resulting interference term in the cross section vanishes as long as one integrates over the azimuthal angle between the lepton and the hadron plane. It is then possible to extract the DVCS cross section by subtracting the Bethe-Heitler contribution, as done by H1.

A new high statistics analysis of DVCS has been performed by the H1 experiment in the kinematic region $4<Q^{2}<80 \mathrm{GeV}^{2}, 30<W<140 \mathrm{GeV}$ and $|t|<1 \mathrm{GeV}^{2}$, using data taken during the year 2004. The $\gamma^{*} p \longrightarrow \gamma p$ cross section has been measured as a function of $Q^{2}$ and as a function of $W^{22}$. The $W$ dependence can be parametrised as $\sigma \propto W^{\delta}$, yielding $\delta \simeq 1.0$ at $Q^{2}=8 \mathrm{GeV}^{2}$, i.e. a value similar to $J / \psi$ production indicating the presence of a hard scattering process. For the first time at HERA II, the DVCS cross section has been measured differentially in $t$ and the observed fast decrease with $|t|$ can be described by the form $e^{-b|t|}$ with $b=5.83 \pm 0.27 \pm 0.50 \mathrm{GeV}^{2}$ at $Q^{2}=8 \mathrm{GeV}^{2}$ (see Fig. 10). The observed t-slope of DVCS is substantially larger than the gluon GPD slope.

This maybe due to a larger radius of the quark transverse distribution at intermediate $Q^{2}$ due to the pion cloud contribution ${ }^{26}$. This effect would be amplified by a factor of $\sim 2$ for the DVCS slope as the gluon GPD contribution as calculated in the NLO ${ }^{27}$ enters with negative sign with weight $\sim 0.5$.

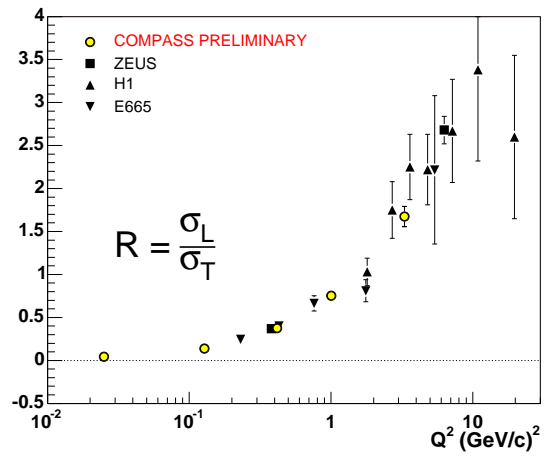

Figure 9. $Q^{2}$ dependence of the ratio $\mathrm{R}$ between the longitudinal and transverse cross section, derived from elastic $\rho^{0}$ mesons production as measured by COMPASS.

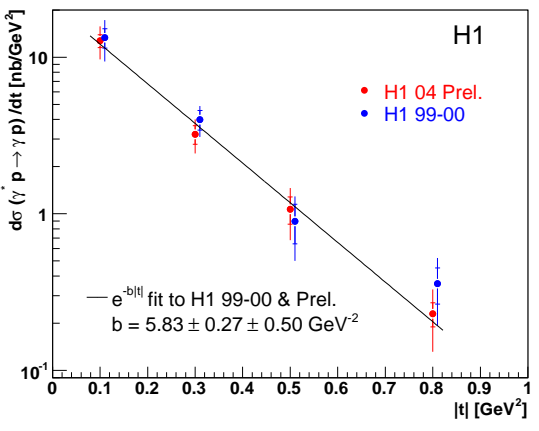

Figure 10. DVCS cross section differential in $t$ at $Q^{2}=8 \mathrm{GeV}^{2}$.

As discussed above, experiments at HERA collider are well designed to study mainly the gluon GPDs at very small $x_{B j}\left(\leq 10^{-2}\right)$. The ex- 
perimental program using COMPASS at CERN (at 100 and/or $190 \mathrm{GeV}$ ) will enlarge the kinematical domain to a large range of $Q^{2}$ and $x_{B j}(1.5$ $\leq Q^{2} \leq 7 \mathrm{GeV}^{2}$ and $\left.0.03 \leq x_{B j} \leq 0.25\right)$, from the process $\mu p \rightarrow \mu^{\prime} p^{\prime} \gamma$. COMPASS is the unique place to measure the azimuthal distribution of the Beam Charge Asymmetry which seems very promising to test the geometrical interpretation of GPDs ${ }^{28}$.

\section{Towards LHC}

In recent years, the production of the Higgs boson in diffractive $p p$ collisions has drawn more and more attention as a clean channel to study the properties of a light Higgs boson or even discover it. This is an interesting example of a new theoretical challenge: to adapt and apply the techniques for the QCD description of diffraction in ep collisions to the more complex case of $p p$ scattering at the LHC. We have already shown in previous sections how first results on hard diffraction can be transmitted from HERA to TEVATRON. The experimental interests to study diffractive processes at the LHC, in connection with the proposal to add forward proton detectors, have grown in parallel with the theory. Various aspects of physics with forward proton tagging at the LHC have been under discussion in our working group ${ }^{10,29}$. In Ref. ${ }^{29,30}$, the unique physics potential of forward proton tagging at $420 \mathrm{~m}$ at the LHC have been presented and discussed.

In Ref. ${ }^{10}$ inclusive and exclusive models have been discussed. Experimentally at LHC, for exclusive events, the full energy available in the center of mass is used to produce the heavy object (dijets, Higgs, diphoton, $W \ldots$... For inclusive events, only part of the available energy is used to produce the heavy object diffractively. In Ref. ${ }^{10}$, it is assumed that the Pomeron is made of quarks and gluons (with the gluon and quark densities deduced from the HERA measurements in shape and the normalisation from TEVATRON data). Then, a quark or a gluon from the Pomeron is used to produce the heavy state. In this context, exclusive model appear to be the limit where the gluon in the Pomeron is a $\delta$ distribution, with no Pomeron remnants for exclusive events. It is shown ${ }^{10}$ that this distinction is quite relevant for experimental applications.

Theoretical calculations of exclusive processes were reviewed in ${ }^{30}$. The survival probability due to the soft interactions of 0.026 was reported and it was argued that the perturbative effects suggested by Bartels et al ${ }^{31}$ are stongly overestimated. Predictions for a wide range of exclusive channels were presented which could be checked already at the TEVATRON collider. 
In ${ }^{32}$ that the t-slope of the gluon GPD in the kinematics relevant for the calculation of the exclusive Higgs production at LHC is substantially smaller than the one assumed by ${ }^{30}$. Using such a smaller slope would result within the model of ${ }^{30}$ to reduction of the gap survival probability by a factor of $\sim 3$. Also it was suggested in ${ }^{32}$ that by measuring the "diffraction pattern," of the $p_{t}$ dependence of the scattered protons one can perform detailed tests of the interplay of hard and soft interactions, and even extract information about the gluon GPD in the proton from the data.

Perspectives of the studies of hard photon- proton (nucleus) interactions at LHC using ultraperipheral collisions of protons (nuclei) with nuclei were reviewed in ${ }^{33}$. It was demonstrated that such measurements would allow to extend the measurements of exclusive production of heavy mesons as compared to HERA to $W \sim 1 T e V$ and allow to measure the gluon densities down to $x \sim 10^{-4}$ for $p_{t} \sim 6 \mathrm{GeV}$. The same measurements would allow to check the prediction that even for large virtualities probability of the diffractive events for which nucleus would remain intact will be of the order 0.2 .

\section{Conclusions}

The findings presented at the session provided further constrains on the interplay between soft and hard physics in the diffraction processes. The forthcoming results from the data analyses of the recent data and the future run at lower energy will allow to move further in resolving open questions such as the dependence of $\alpha^{\prime}$ on the hardness scale, contribution of higher twist processes in the inclusive and exclusive diffraction, energy dependence of the exclusive processes at high resolution scales.

Coordinators would like to thank the participants of the session as well as J.Bartels, A.Freund and M.Ryskin for numerous contributions and inputs.

\section{References}

1. J. C. Collins, Phys. Rev. D 57, 3051 (1998) [Erratum-ibid. D 61, 019902 (2000)] [arXiv:hep-ph/9709499].

2. M. Soares, these proceedings.

3. L. Frankfurt, W. Koepf and M. Strikman, Phys. Lett. B 405, 367 (1997) [arXiv:hep-ph/9702236].

4. A.B. Kaidalov, V.A. Khoze, A.D. Martin, M.G. Ryskin,

5. S. Kumano, Phys. Rept. 303, 183 (1998) [arXiv:hep-ph/9702367]. 
6. H1 Coll., A. Aktas et al., sumitted to Eur. Phys. J. C; hep-ex/0606003.

7. H1 Coll., A. Aktas et al., sumitted to Eur. Phys. J. C; hep-ex/0606004.

8. E. Sauvan, these proceedings.

9. ZEUS Coll., S. Chekanov et al., Nucl. Phys. B 713 (2005) 3; hep-ex/0501060.

10. C. Royon, these proceedings.

11. G. Watt, these proceedings.

12. M. Mozer, these proceedings.

13. A. Bonato, these proceedings.

14. C. Royon, L. Schoeffel, R. Peschanski and E. Sauvan, Nucl. Phys. B 746 (2006) 15 [arXiv:hep-ph/0602228].

15. M. Gallinaro, these proceedings.

16. B. Cox, J. Forshaw, Comput. Phys. Commun. 144, 104 (2002). POMWIG implements the diffractive collisions, using all hard sub-processes, into the HERWIG Monte Carlo generator.

17. M. Boonekamp and T. Kucs, Comput. Phys. Commun. 167, 217 (2005); DPEMC extends the POMWIG Monte Carlo generator to include new models of central production through inclusive and exclusive Double Pomeron Exchange in hadron collisions.

18. J. Olson, these proceedings.

19. J. C. Collins, L. Frankfurt and M. Strikman, Phys. Rev. D 56, 2982 (1997) [arXiv:hep-ph/9611433].

20. N. d'Hose, these proceedings.

21. M. Diehl, these proceedings.

22. B. Roland, these proceedings.

23. H. Kowalski, these proceedings.

24. S. J. Brodsky, L. Frankfurt, J. F. Gunion, A. H. Mueller and M. Strikman, Phys. Rev. D 50, 3134 (1994) [arXiv:hep-ph/9402283].

25. L. Frankfurt, W. Koepf and M. Strikman, Phys. Rev. D 57, 512 (1998) [arXiv:hep-ph/9702216].

26. M. Strikman and C. Weiss, Phys. Rev. D 69, 054012 (2004) [arXiv:hep$\mathrm{ph} / 0308191]$.

27. A. Freund and M. F. McDermott, Phys. Rev. D 65, 091901 (2002) [arXiv:hep$\mathrm{ph} / 0106124]$.

28. F.H. Heinsius, these proceedings.

29. B. Cox, these proceedings.

30. A.D. Martin, these proceedings.

31. J. Bartels, S. Bondarenko, K. Kutak and L. Motyka, "Exclusive Higgs boson production at the LHC: Hard rescattering Phys. Rev. D 73, 093004 (2006) [arXiv:hep-ph/0601128].

32. C. Hyde-Wright, these proceedings.

33. M. Strikman, these proceedings. 